\title{
Kostas Karyotakis, le poète-« phénomène » de la génération dite « des années 1920 » en Grèce
}

Questions de conscience et de poésie

Kostas Karyotakis, The Poet-"Phenomenon" of the Generation known as "1920's

Generation" in Greece: Matters of Conscience and Poetry

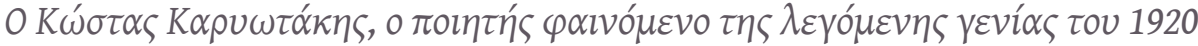

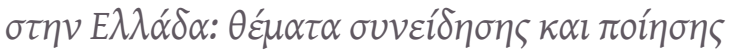

\section{Vassiliki Tsaita-Tsilimeni}

\section{OpenEdition}

\section{Journals}

Édition électronique

URL : https://journals.openedition.org/ceb/5491

DOI : 10.4000/ceb.5491

ISSN : 2261-4184

Éditeur

INALCO

Édition imprimée

Date de publication : 1 mars 2015

ISBN : 978-2-85831-224-5

ISSN : 0290-7402

Référence électronique

Vassiliki Tsaita-Tsilimeni, « Kostas Karyotakis, le poète-« phénomène » de la génération dite « des années 1920 » en Grèce », Cahiers balkaniques [En ligne], Hors-série | 2015, mis en ligne le 11 décembre 2015, consulté le 06 juillet 2021. URL : http://journals.openedition.org/ceb/5491 ; DOI : https://doi.org/10.4000/ceb.5491

Ce document a été généré automatiquement le 6 juillet 2021

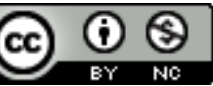

Cahiers balkaniques est mis à disposition selon les termes de la Licence Creative Commons Attribution - Pas d'Utilisation Commerciale 4.0 International. 


\section{Kostas Karyotakis, le poète-« phénomène » de la génération dite « des années 1920 » en Grèce}

Questions de conscience et de poésie

Kostas Karyotakis, The Poet-"Phenomenon" of the Generation known as "1920's

Generation" in Greece: Matters of Conscience and Poetry

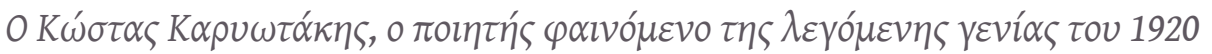

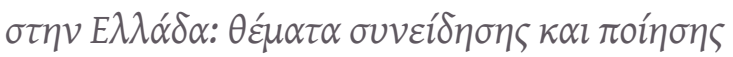

Vassiliki Tsaita-Tsilimeni

1 Dès le début de notre étude, il est très important de souligner que Karyotakis est un cas très différent de tous les autres poètes de sa génération pour plusieurs raisons qui ne concernent pas seulement la thématique de sa poésie, mais aussi plusieurs aspects de son caractère, de son style poétique, de sa pensée. Il s'agit d'un poète qui réalise un vrai changement dans la poésie de son époque en créant son propre style qui marque une génération entière, qui influence plusieurs poètes après sa mort et qui possède des caractéristiques nouvelles pour l'époque quant à la nature de la poésie.

\section{Un cas unique}

2 Agras écrit ${ }^{1}$ dans sa critique de Karyotakis qu'il s'agit d'un poète qui est tourné vers un réalisme assez dur. Sa poésie, surtout ses premiers recueils, témoigne d'une nature mélancolique et pessimiste, mais il s'agit plutôt d'une mélancolie qui se tourne vers un réalisme strict, contre ce qui constitue sa quotidienneté. Il est vrai que sa poésie évolue beaucoup de recueil en recueil. Les deux premiers recueils sont plus proches du climat du romantisme; ils sont caractérisés par un ton assez nostalgique et amer en ce qui concerne les sujets de la mort, de l'amour, de la poésie, etc. Après ces deux recueils, 
Karyotakis opère un grand changement, et sa poésie acquiert la nature qui le caractérisera par la suite. Agras se réfère aussi à un autre aspect de sa poésie, le fait que le poète n'ait emprunté à aucun autre, plus précisément, il écrit qu'« il a pris le contenu de sa poésie directement en lui-même $»^{2}$.

3 Karyotakis est un poète qui affiche une voix poétique pure dès ses premiers écrits. Sa poésie est plus directe en ce qui concerne sa tonalité et il crée un style assez nouveau pour son époque en ayant bien sûr, comme tous les autres poètes de sa génération, été influencé par les poètes étrangers, comme Baudelaire, Rimbaud, Villon, Heine, Toulet, etc. En général, le nouveau style que crée Karyotakis dans la poésie grecque de l'époque se caractérise par la satire, l'ironie et les attaques contre les éléments pourris d'une société tourmentée.

4 Karyotakis est presque le seul poète grec de cette génération qui ait, par moments, une conscience précise de cette sensation de tourment qui le touche lui-même ainsi que ses contemporains ; cette sensation naît des questions qu'ils se posent sur leur rôle dans la société comme poètes, sur les chances de la Grèce comme nation hellénique en ces années si troublées, sur leur nature tourmentée d'êtres humains qui essaient de survivre dans une époque si dure, marquée par plusieurs stigmatisations sociales, et sur la limitation de leurs libres choix, choix qui pourraient les conduire à une liberté personnelle plus claire et plus précise.

5 Autrement dit, en lisant l'œuvre de Karyotakis, on voit toute la thématique obscure et cette nature mélancolique et pessimiste qui caractérise la génération de 1920, mais, en même temps, on rencontre une voix claire, consciente, originale et remarquablement audacieuse pour son époque. Il se trouve à un niveau plus élevé que les autres et il peut distinguer assez clairement la tragédie profonde de sa génération et les raisons qui l'ont provoquée. Il a une pensée philosophique puissante qui lui permet d'écrire une poésie dynamique qui reflète dans sa vérité son époque obscure. Autrement dit, la poésie de Karyotakis est le miroir le plus exact de la crise de son époque.

6 À la différence des autres poètes de sa génération, Karyotakis a les yeux ouverts, il a une pleine conscience, malgré les grandes difficultés et l'atmosphère confuse de son présent. Les autres vivent dans cette atmosphère et ils utilisent souvent la poésie comme seule manière d'expression, mais ils ne pensent pas vraiment à sa force, ils ne voient pas clairement son rôle et ses possibilités comme une arme importante dans une société qui vit dans une grande relativité et en même temps dans une grande incertitude; ils ne voient pas non plus les possibilités de l'homme dans une période de guerres et de changements multiples. Tous les autres poètes qui constituent la génération de 1920 perçoivent comme un changement dans la poésie cette atmosphère lourde qui pèse sur la plupart de leurs rêves irréalisables et sur leur présent impuissant à leur offrir le bonheur et la quiétude. Par rapport à Karyotakis, bien qu'ils aient aussi, sans doute, leurs éclairs philosophiques et une conscience importante et non négligeable du réel, ils n'arrivent pas à percevoir la vraie réalité dans laquelle ils vivent et à voir consciemment la poésie comme un moyen pour réussir à exprimer quelque chose d'autre que leurs plaintes et leurs vies apparemment « immobiles».

7 Karyotakis, par rapport aux autres poètes, ses contemporains, vit un réveil dur et tourmenté de la conscience. De plus, en ce qui concerne la dernière partie de sa poésie, celle des satires, on peut prétendre qu'il s'apparente à la crise proche, au sens de la réaction face à une régularité en effet, dans le cas de la génération de 1920, cette régularité se traduit par une certaine convention dans une atmosphère décadente qui 
provient soit des événements graves de leur époque, soit de l'imitation de modèles de la poésie étrangère, soit de la faiblesse de la poésie grecque à trouver sa propre identité. Autrement dit, on peut parler d'une sorte d'oscillation de Karyotakis (comme poète et comme homme de son époque) entre sa vie précise dans une époque précise et la formation de sa conscience sur sa génération poétique et sociale.

\section{Recueil par recueil}

En examinant son œuvre recueil par recueil, on commence par son premier recueil qui

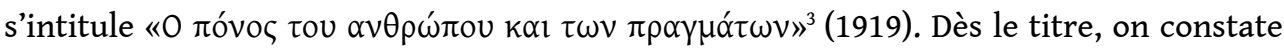
une forte sensibilité du poète face aux chagrins des hommes et des choses. Le titre témoigne d'une conscience qui se forme peu à peu sur les choses du monde, tout en portant attention à ses peines. Ce premier recueil de Karyotakis est constitué d'un petit nombre de poèmes (dix), dont la minorité concerne un « ego " qui décrit avec clarté sa nature mélancolique, tandis que la majorité se réfère à un "nous " qui nous prouve que, dès ses 23 ans, ce jeune poète porte un regard clair sur sa génération. Le jeune poète en ouvrant les yeux, est à l'écoute de la génération à laquelle il appartient, et il choisit de parler simplement de tous ceux qu'il observe en se sentant en même temps fortement seul, peut-être parce qu'il constate sa différence avec tous les autres, ses contemporains.

En commençant ses premiers pas poétiques, Karyotakis arrive à décrire par des vers simples et purs certains aspects de la tragédie de l'individu dans cette période difficile

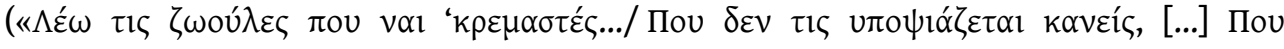

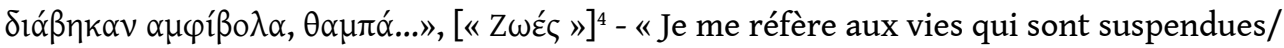
Que personne ne soupçonne, [...] Qui passèrent aléatoirement, indéfiniment... »), tout en sachant que lui-même est aussi un individu identique dans la collectivité de sa génération.

10 Son imagination troublée dont Agras a beaucoup parlé5 lui permet d'atteindre plus facilement les fondements les plus profonds d'un réalisme qui deviendra plus tard absolument extrême et qui le conduira à un pessimisme également extrême. Karyotakis lui-même se réfère à sa curiosité brûlante et à son imagination malsaine comme deux

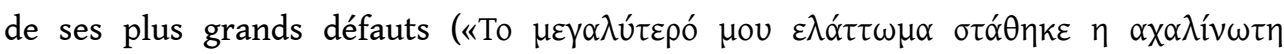

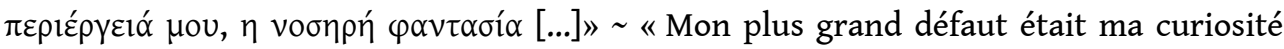
brûlante, mon imagination malsaine [...] ») . Peut-être ces deux défauts sont-ils aussi les deux raisons fondamentales pour lesquelles sa perception des choses suivit une route beaucoup plus consciente que celle de ses contemporains. Sa forte curiosité pour explorer profondément la nature réelle de son époque, alliée à une imagination proche du climat pessimiste de ces années, conduisirent le poète d'un côté à avoir un regard sur les choses plus profond et, de l'autre, à avoir un caractère solitaire et une nature personnelle plus oscillante et plus troublée, dès le moment où il a constaté le malheur de son époque. Ce malheur est dû au besoin fort des poètes de chercher un monde où le bonheur existerait et où la quotidienneté si dure n'existerait plus, il est dû également à leur conception de la poésie, comme presque condamnée à n'exprimer que leurs tourments sans pouvoir les aider à créer le monde différent dont ils rêvent. La différence de Karyotakis, c'est qu'il a passablement changé la poésie de son époque en reflétant dans ses vers la réalité du monde dans lequel il vivait aussi lui-même, en examinant plus attentivement la poésie étrangère et ses buts, et en utilisant de 
nouvelles formes poétiques qui ont clairement donné naissance à une poésie d'une force différente, et d'un caractère assez diachronique.

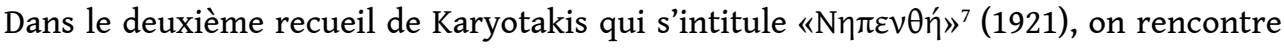
un « ego » qui se tourmente dans une amertume immense, qui se sent plus seul encore, qui a une conscience de la " nécessité » même de la nature de son époque, de sa propre nature et de l'isolement de lui-même par les autres, et de son âme dans le monde général et le temps.

Comme exemple, on peut prendre, dans le deuxième recueil de Karyotakis, le poème

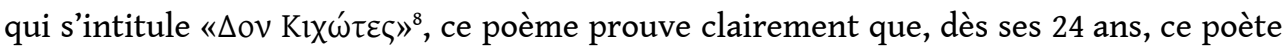
a une conscience très forte de la nature poétique et personnelle de ses contemporains

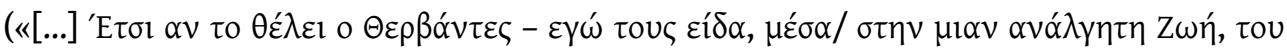

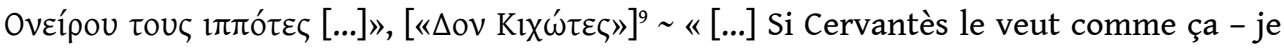
les vis, dans/la Vie indifférente, les chevaliers du Rêve [...]»), leur effort vain de

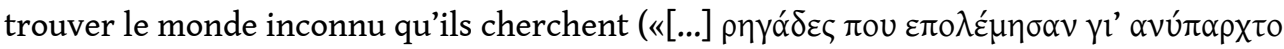

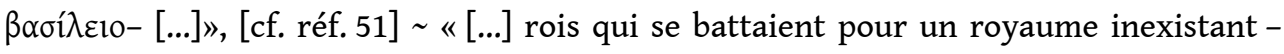

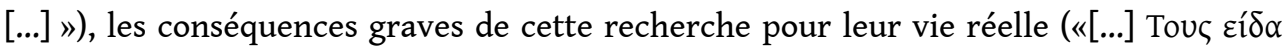

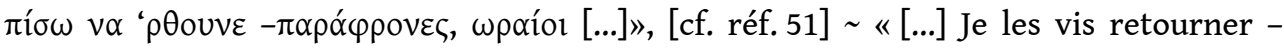
déments, jolis [...]») et à la fin, en ce qui concerne l'empreinte que laissera cette recherche vaine et ses résultats sur la vie et la poésie des poètes de son époque, une empreinte qui apparaîtra à la génération qui suit comme "une blessure vaine au

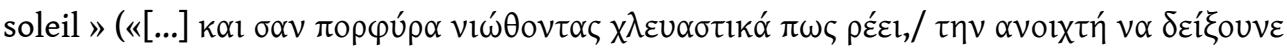

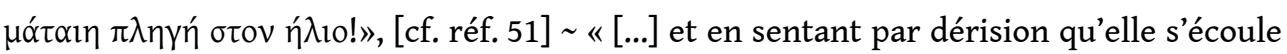
comme la pourpre,/de montrer l'ouverte blessure vaine au soleil!»). Autrement dit, Karyotakis est très en avance sur son époque, tout en étant en même temps victime de son époque dans sa vie et ses malheurs.

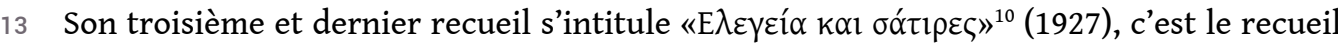
le plus dur, et le plus extrême du poète. Sa maturité est plus aiguë et elle est influencée par le grand pessimisme qui le tourmente. On ne doit pas oublier aussi qu'en 1922, cinq ans avant la publication de ce recueil, Karyotakis apprit qu'il était malade de la syphilis. C'est dans ce recueil qu'il écrit ses satires, une œuvre dont on a beaucoup parlé jusqu'à nos jours pour sa langue dure, sa nature assez déprimante et son lien possible avec le suicide de Karyotakis.

Dans ce recueil, il y a beaucoup de poèmes qui se réfèrent à un « ego » qui semble avoir revu sa vie, et en avoir déduit sa nature tragique dans l'époque où il vit. Il note l'aggravation de son monde intérieur pessimiste, il voit la dissipation totale de ses espoirs, il conserve sa clarté sur le destin tragique de sa génération, il voit devant lui sa mort, il continue de constater l'ignorance générale du monde, il continue de constater l'impasse absolue de sa génération, sa solitude, le fait qu'elle déteste son seul recours: la poésie, sa vie tragique qui semble rester dans l'inexistence. Dans ce recueil, Karyotakis utilise ses armes les plus pointues et il n'hésite pas à écrire des vers qui reflètent la tragédie d'une époque, les oscillations de la pensée individuelle, les grandes questions qui tourmentent lui-même et ses contemporains, et finalement sa propre tragédie : l'absence générale de sentiment. 


\section{Une génération en proie aux tourments}

15 En plus, il acquiert une conscience presque absolue du fait que sa génération semble

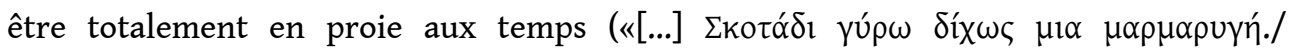

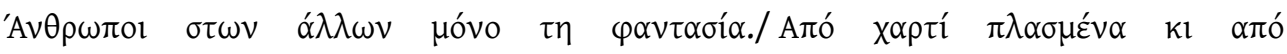

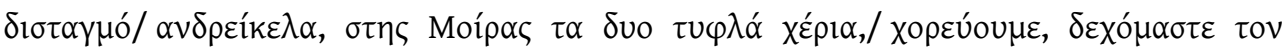

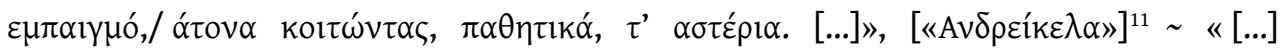
Obscurité autour sans un scintillement./Nous existons seulement dans imagination des autres./faits par papier et par hésitation/pantins, dans les deux mains du Destin,/nous dansons, nous acceptons la moquerie,/en voyant languissamment, passivement, les étoiles. [...]»). Agras écrit dans son étude critique sur Karyotakis: "Quel sentiment attend l'homme, s'il continue de vivre et ne s'afflige pas, après la déception définitive? La satire. $\rrbracket^{12}$. Il est vrai que les satires de Karyotakis regroupent 16 poèmes assez forts qui se réfèrent à la syphilis ${ }^{13}$, à la liberté inexistante ${ }^{14}$, à la poésie d'A. Kalvos ${ }^{15}$, à la répulsion du poète pour les poupées ${ }^{16}$, à l'ambition des poètes ${ }^{17}$, à la vie tragique des fonctionnaires dans son époque ${ }^{18}$, à l'avilissement de l'individu par la politique et la société $^{19}$, etc. Dans les satires, on voit clairement la réaction de Karyotakis contre toutes ces choses qui constituent et caractérisent son époque.

Dans le dernier recueil de Karyotakis, on constate plus clairement que dans les autres deux recueils précédents sa nature divisée entre d'une part sa prise de conscience forte de sa génération, ses tourments et son ignorance et, d'autre part, sa vie personnelle plongée dans la même atmosphère et réalité de son époque et qui formait sa conscience en alliance avec sa curiosité brûlante et son imagination malsaine. Par conséquent, on peut dire sans hésitation que la crise de son époque se reflète dans son œuvre.

17 Karyotakis est le seul poète de la génération de 1920 qui parvient à s'approcher au plus près de la création d'un nouveau visage de la poésie. En effet, dès le moment où il reflète dans ses vers toute la crise de son époque, il utilise les symboles d'une manière proche des formes authentiques de l'étranger sans rester néanmoins dans l'imitation et, finalement, il écrit des satires qui incarnent une véritable attaque de la société de son époque.

Karyotakis, en étant d'une certaine manière le miroir poétique de son époque, voit clairement que la poésie de cette époque, bien qu'elle soit plongée dans une atmosphère lourde et souvent inchangée, représente un pas important et intermédiaire qui conduira un jour à une formation poétique nouvelle. Il voit que cette poésie a un corps uni malgré les tourments que lui-même n'omet pas de mettre en évidence. Il faut remarquer qu'il nous offre dans sa poésie la réflexion des divers niveaux de la poésie de

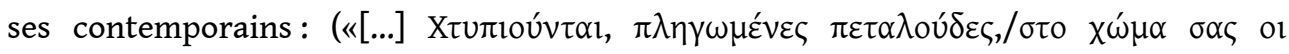

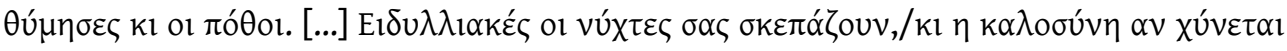

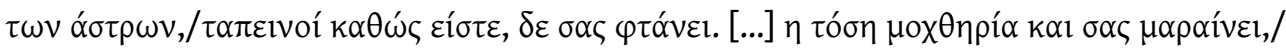

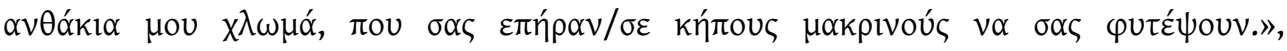
[«Пoı $\left.\tau \varepsilon^{\prime} \zeta »\right]^{20} \sim$ «[...] Ils se battent comme des papillons blessés,/à votre terre les souvenirs et les désirs. [...] Les nuits idylliques vous recouvrent,/et si la bonté des étoiles se déverse,/humbles comme vous êtes, elle ne vous atteint pas. [...] tant de malignité vous flétrit,/mes petites fleurs pâles, qu'on a prises/pour vous planter dans de lointains jardins. »). Il réussit à créer une nouvelle poésie qui a sa propre place dans une génération qui souffre pour plusieurs raisons (politiques, sociales, etc.), et ainsi, il 
parvient à montrer aussi l'évolution existante dans la poésie de son époque qui, à première lecture, semble presque figée.

\section{Conclusion}

Karyotakis incarne donc une conscience poétique sur la poésie en général et sur la poésie hellénique de son époque ; d'un côté, il reflète à travers ses vers la crise générale de son époque, et de l'autre, à travers de ce reflet, il réussit finalement à créer une voix poétique très authentique de son époque.

Par conséquent, sa crise de la conscience poétique n'est qu'une étape intermédiaire et nécessaire jusqu'au moment où il écrit directement, d'une voix claire, la poésie la plus caractéristique de son époque.

\section{BIBLIOGRAPHIE}

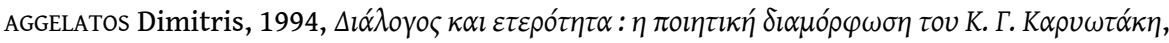

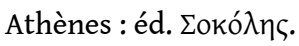

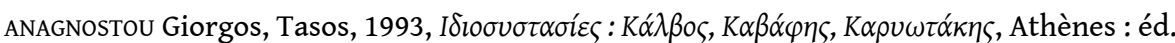

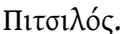

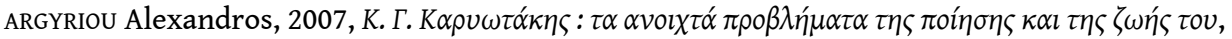

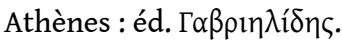

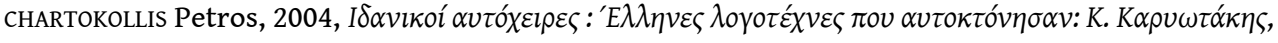

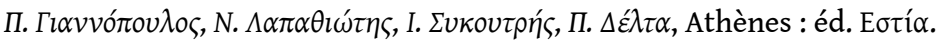

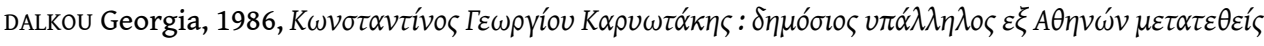

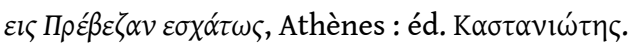

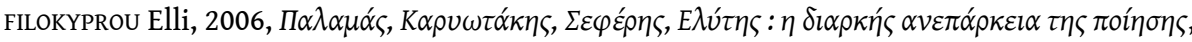

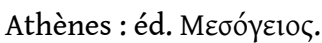

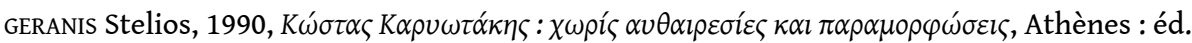

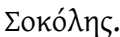

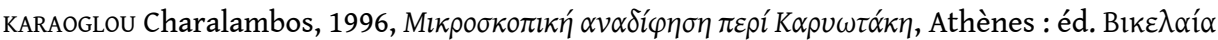

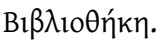

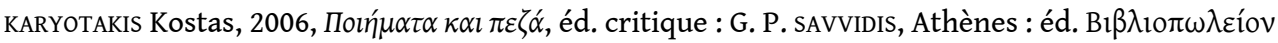

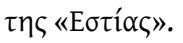

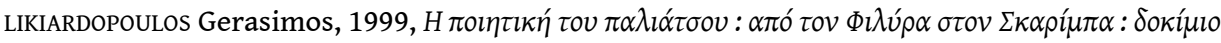

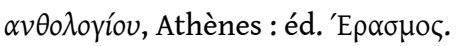

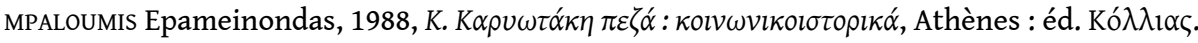




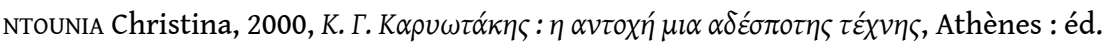

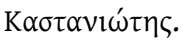

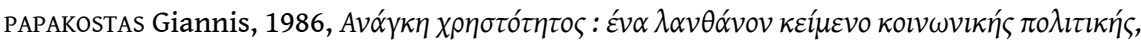

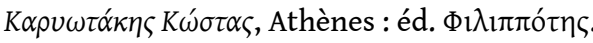

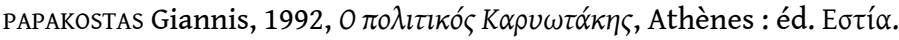

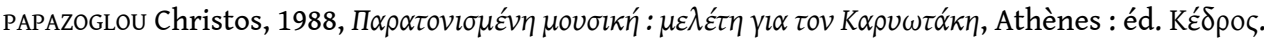

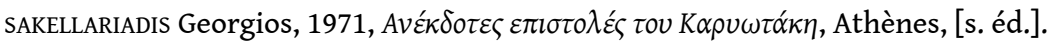

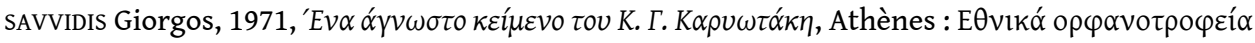

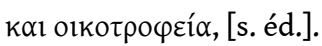

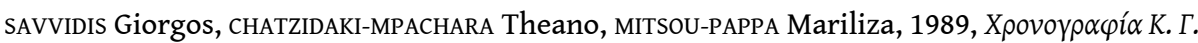

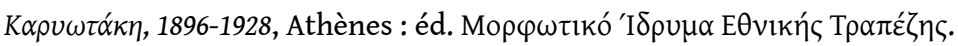

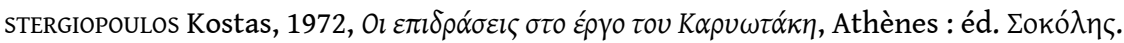

\section{NOTES}

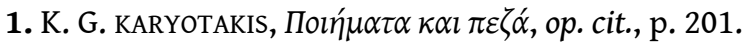

2. Ibid., p. 198.

3. K. G. KARYOTAKIS, Пoı́́ $\mu \alpha \tau \alpha \kappa \alpha l \pi \varepsilon \zeta \alpha ́$, op. cit., p. 3-16.

4. Ibid., p. 7.

5. Ibid., p. 195.

6. Cf. Kostas KARYOTAKIS (Wikipedia).

7. K. G. KARYOTAKIS, Пoเท́ $\mu \alpha \tau \alpha \kappa \alpha l \pi \varepsilon \zeta \alpha$, op. cit., p. 17-54.

8. Ibid., p. 22-23.

9. Ibid., p. 23.

10. Ibid., p. 61-115.

11. Ibid., p. 89.

12. Ibid., p. 203.

13. Ibid., p. 107.

14. Ibid., p. 98.

15. Ibid., p. 99-101.

16. Ibid., p. 102.

17. Ibid., p. 103.

18. Ibid., p. 104.

19. Ibid., p 105 et 106.

20. K. G. KARYOTAKIS, Пoıฑ́ $\mu \alpha \tau \alpha \kappa \alpha l \pi \varepsilon \zeta \alpha$, op. cit., p. 26. 


\section{RÉSUMÉS}

Dans notre communication, nous tenterons de présenter et d'analyser les raisons pour lesquelles Kostas Karyotakis, poète grec de la " génération de 1920 ", est un cas très différent de tous les autres poètes de sa génération. Ces raisons ne concernent pas seulement la thématique de sa poésie, mais aussi plusieurs aspects de son caractère, de son style poétique et de sa pensée.

En lisant l'œuvre de Karyotakis, on voit toute la thématique obscure et la nature mélancolique et pessimiste qui caractérise la génération de 1920, mais, en même temps, on rencontre une voix claire, consciente, originale et remarquablement audacieuse pour son époque. Parfois, on a l'impression que Karyotakis se trouve à un niveau plus élevé que les autres et on peut distinguer chez lui clairement la tragédie profonde de sa génération et les raisons qui l'ont provoquée.

Karyotakis est presque le seul poète grec de cette génération qui ait, par moments, une conscience précise du tourment qui le touche lui et ses contemporains ; cette sensation naît des questions qu'ils se posent sur leur rôle dans la société comme poètes, sur les chances de la Grèce comme nation hellénique en ces années si troublées (1897-1930), sur leur nature tourmentée d'humains qui essaient de survivre dans une époque si dure marquée par plusieurs stigmatisations sociales, et sur la limitation de leurs libres choix, choix qui pourraient les conduire à une liberté personnelle plus claire et plus précise.

In our communication, we try to present and analyse the reasons why Kostas Karyotakis, Greek poet of the "1920's" generation, is a very different case from the other poets of his generation. These reasons do not concern just the theme of his poetry, but also many aspects of his character, his poetic style, his thought, etc.

In other words, by reading the work of Karyotakis, we see all his dark theme and his melancholic and pessimist nature that characterize the 1920's generation and, in the same moment, we have a clear, conscious, original and remarkably bold view about his time. Sometimes we get the antithetical impression that Karyotakis is at a higher level than the others and can clearly distinguish the deep tragedy of his generation and the reasons that caused it.

Karyotakis is probably the only poet of this generation who has, at times, a clear awareness of the sensation of the torment that touches himself and his contemporaries; sensation that raises questions about their role in the society as poets, about the odds of Greece as Hellenic nation in these troubled times (1897-1930), about their turbulent nature of being a human being who is trying to survive in an era which was marked by so many social stigmas, and about the limitation of their free choice, choice that would lead to a clearer and more precise personal freedom.

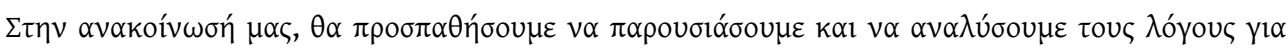

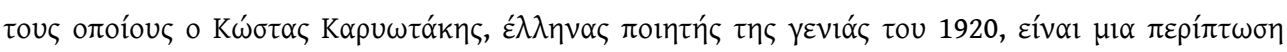

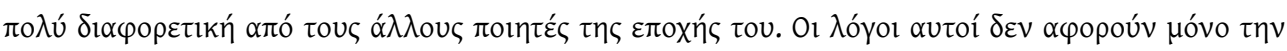

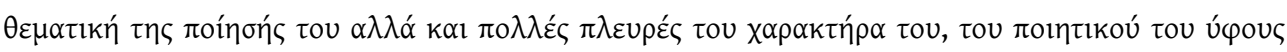

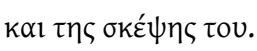

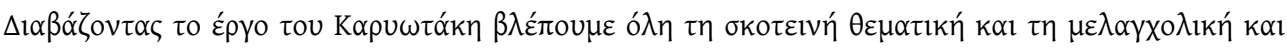

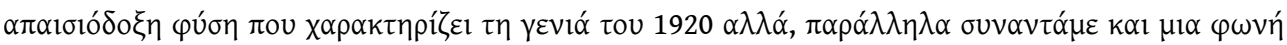

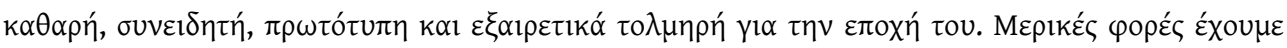

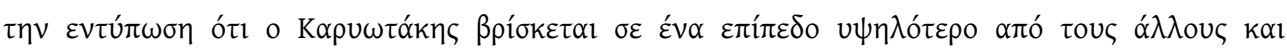

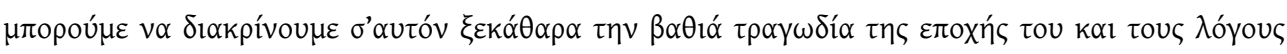

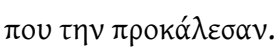

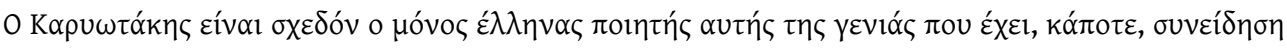

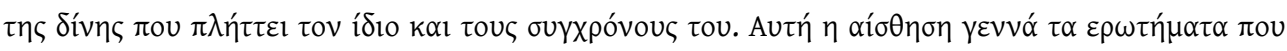




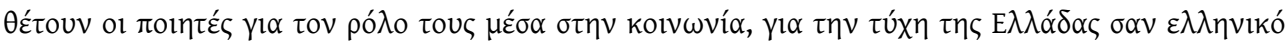

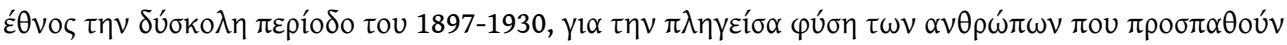
$v \alpha \varepsilon \pi ı$ ı

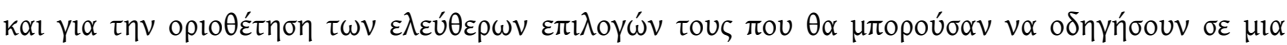

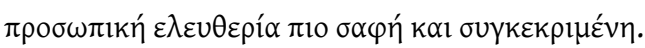

\section{INDEX}

Index géographique : Grèce

Mots-clés : Karyotakis Kostas (1896-1928), Karyotakis Kostas (1896-1928), génération de 1920

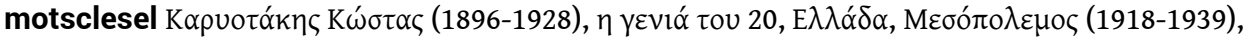

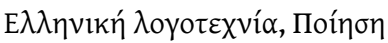

motsclestr Karyotakis Kostas (1896-1928), Nesil 1920, Yunanistan, Savaşlar Arasında (1918-1939),

Yunan edebiyatı, şiir

motsclesmk КОСТАС КАРУОТАКИС (1896-1928), ГЕНЕРАЦИЈА 1920, ГРЦИЈА, ПОМЕЃУ ВОЈНИТЕ, ГРУКАТА ЛИТЕРАТУРА, ЛОЕЗИЈА

Keywords : Karyotakis Kostas (1896-1928), 1920' generation, Greece, Interwar (1918-1939), Greek Literature, poetry

Thèmes : Littérature grecque, Poésie

Index chronologique : entre-deux-guerres (1918-1939)

\section{AUTEUR}

VASSILIKI TSAITA-TSILIMENI

Université de Genève 